\title{
Hepatic Encephalopathy: An Update on the Pathophysiology and Therapeutic Options
}

\author{
Saleh Elwir* and Robert S. Rahimi \\ Annette C. and Harold C. Simmons Transplant Institute, Baylor University Medical Center, Dallas, TX, USA
}

\begin{abstract}
Hepatic encephalopathy is a spectrum of reversible neuropsychiatric abnormalities, seen in patients with liver dysfunction and/or portosystemic shunting. One of the most debilitating complications of cirrhosis, encephalopathy affects $30-45 \%$ of cirrhotics. In addition to significantly affecting the lives of patients and their caregivers, it is also associated with increased morbidity and mortality as well as significant utilization of health care resources. In this paper, we provide an overview on the pathophysiology, diagnosis, management and newer therapies of hepatic encephalopathy.

Citation of this article: Elwir S, Rahimi RS. Hepatic encephalopathy: an update on the pathophysiology and therapeutic options. J Clin Transl Hepatol 2017;5(2):142-151. doi: 10. 14218/JCTH.2016.00069.
\end{abstract}

\section{Introduction}

Cirrhosis is the $12^{\text {th }}$ leading cause of death in the United States (US), accounting for more than 36,000 deaths and having a death rate of 11.5 per 100,000 in $2013 .{ }^{1}$ Hepatic encephalopathy (HE) is one of the most debilitating complications of cirrhosis and severely affects the lives of patients and their caregivers. ${ }^{2} \mathrm{HE}$ embodies a spectrum of reversible neuropsychiatric abnormalities, which are seen in patients with liver dysfunction and/or portosystemic shunting. ${ }^{3-6}$

$\mathrm{HE}$ is generally divided into covert and overt types. Covert $\mathrm{HE}$ includes minimal $\mathrm{HE}$ and grade $1 \mathrm{HE}$ according to the West Haven criteria (WHC), while overt HE includes grades 2-4 (Table 1). ${ }^{3} \mathrm{HE}$ is further divided into 3 subtypes based on etiology, with type A associated with acute liver failure, type $B$ with portosystemic shunts (such as transjugular intrahepatic portosystemic shunt (TIPS)) and type $C$ with cirrhosis. ${ }^{4,6}$ This

Keywords: Hepatic encephalopathy; Cirrhosis; Lactulose; Rifaximin.

Abbreviations: AMS, altered mental status; BCAAs, branched chain amino acids; CFF, Critical flicker frequency; CT, Computed tomography; EEG, electroencephalogram; GABA, gamma-amino butyric acid; GPB, glycerol phenylbutyrate; $\mathrm{HE}$, hepatic encephalopathy; HR, hazard ratio; LOLA, L-ornithine-L-aspartate; MARS, molecular adsorbent recirculating system; MELD, model for end-stage liver disease; $\mathrm{NH}_{3}$, Ammonia; NNS, number needed to scan; OP, ornithine phenylacetate; PAA, phenylacetate; PAGN, urinary phenylacetylglutamine; PEG, polyethylene glycol; PPIs, proton pump inhibitors; RCT, randomized controlled trial; SMT standard medical therapy; TIPS, transjugular intrahepatic portosystemic shunt; ULN, upper limit of normal; US, United States; WHC, West Haven criteria.

Received: 24 November 2016; Revised: 11 February 2017; Accepted: 24 March 2017

* Correspondence to: Saleh Elwir, Annette C. and Harold C. Simmons Transplant Institute, Baylor University Medical Center, 3410 Worth Street, Suite 950, Dallas, TX 75246, USA. +1-214-820-8500, Fax: +1-214-820-0993, E-mail: saleh. elwir@bswhealth.org review will focus on $\mathrm{HE}$ types $\mathrm{B}$ and $\mathrm{C}$, which can either be spontaneous or precipitated. Depending on its frequency, HE is classified as episodic (if one episode occurs in 6 months), recurrent (if more than one episode occurs in a 6-month period) or persistent (if the neuropsychiatric abnormalities persist and do not return to baseline). ${ }^{3,4,6}$

\section{Incidence and disease burden}

Overt and clinically evident forms of HE occur in $30-45 \%$ of patients with cirrhosis and in $10-50 \%$ of patients who undergo TIPS placement, while the subtler forms occur in a higher percentage of patients. ${ }^{7} \mathrm{HE}$ is associated with increases in hospitalizations, health care costs and mortality. In 2003 alone, over 40,000 patients were hospitalized in the US for a primary diagnosis of HE. ${ }^{7}$ Resource utilization for patients discharged from US hospitals with a diagnosis of HE increased from 2005 to 2009, with total national charges related to $\mathrm{HE}$ estimated at $\$ 7244.7$ million in 2009.8 Moreover, $\mathrm{HE}$ remains the most common reason for readmission of patients hospitalized with decompensated cirrhosis. ${ }^{9}$

Development of HE is associated with increased mortality. In a study by Bustamante and colleagues, ${ }^{10}$ patients with cirrhosis who developed HE had a 1-year survival probability of $42 \%$ and 3 -year survival probability of $23 \%$. The increased mortality observed in patients with $\mathrm{HE}$ is independent of model for end-stage liver disease (MELD) score. ${ }^{11}$ The presence of grade 3 or $4 \mathrm{HE}$ in patients with cirrhosis awaiting liver transplantation has been reported as associated with a $66 \%$ greater risk of 90 -day mortality, as compared to patients without HE. ${ }^{12}$ Patients who had grade 3 or $4 \mathrm{HE}$ at time of liver transplantation showed significantly lower 1- and 5 -year survival rates, as compared to patients without $\mathrm{HE}$ at time of transplant (1-year: $82.5 \%$ vs $90.3 \%, p<0.001$; 5-year: $69.1 \%$ vs $74.4 \%, p<0.001){ }^{13}$

\section{Pathophysiology}

The pathophysiology of $\mathrm{HE}$ is multifactorial and involves agents such as ammonia $\left(\mathrm{NH}_{3}\right)$, inflammatory cytokines, manganese deposition in the basal ganglia, and benzodiazepinelike compounds (i.e. gamma-amino butyric acid (GABA)); recent studies are also recognizing roles for microbiota and aromatic amino acids. ${ }^{3,5,14}$ Although HE pathogenesis is a complex entity (Fig. 1) with multiple components resulting in functional impairment of neuronal cells-none of which are well understood- $\mathrm{NH}_{3}$ has been considered the primary pathophysiologic mechanism of $\mathrm{HE}$.

$\mathrm{NH}_{3}$ is mostly a gut-derived nitrogenous toxin produced by bacterial metabolism of urea from proteins that are consumed 
Table 1. Grade or stage of HE according to the west haven criteria ${ }^{6,15,34}$

\begin{tabular}{ll}
\hline $\begin{array}{l}\text { Grade or } \\
\text { stage }\end{array}$ & Clinical features \\
\hline 1 & $\begin{array}{l}\text { Minimal lack of awareness, shortened } \\
\text { attention span, impairment of calculation } \\
\text { ability, altered sleep pattern }\end{array}$ \\
2 & $\begin{array}{l}\text { Disorientation (usually to time), lethargy, } \\
\text { inappropriate behavior, personality changes }\end{array}$ \\
3 & $\begin{array}{l}\text { Gross disorientation (time and place), marked } \\
\text { confusion, somnolent but responsive to } \\
\text { stimuli, incomprehensible speech } \\
\text { Coma, unresponsive to verbal or physical } \\
\text { stimulation }\end{array}$ \\
\hline
\end{tabular}

in the human diet. ${ }^{5,14} \mathrm{NH}_{3}$ is metabolized by the liver and subsequently cleared by the kidneys, and to a lesser extent by the muscles. ${ }^{5}$ In patients with cirrhosis, liver dysfunction impairs hepatic metabolism of $\mathrm{NH}_{3}$ and portal hypertension results in shunting of $\mathrm{NH}_{3}$-rich portal blood to the systemic circulation without detoxification. ${ }^{5,14,15}$ In the brain, $\mathrm{NH}_{3}$ crosses the blood-brain barrier and is metabolized in the astrocytes by glutamine synthetase, which converts $\mathrm{NH}_{3}$ and glutamate to glutamine. ${ }^{14}$

Accumulation of glutamine in astrocytes creates an osmotic gradient, resulting in astrocyte swelling ${ }^{15}$ and generation of reactive oxygen species, thereby contributing to the cerebral dysfunction seen in $\mathrm{HE}^{14}$ The dysbiosis and increased gut permeability seen in cirrhosis also results in an increase in the production of multiple inflammatory cytokines, which contributes to an increase in blood-brain barrier permeability and contributes to cerebral edema. ${ }^{3}$ Ammonia also binds to GABA receptors on astrocytes, leading to activation and production of neurosteroids, which further contribute to the occurrence of $\mathrm{HE}{ }^{5}$ The neurotoxicity of $\mathrm{NH}_{3}$ results in increased resting membrane potential and inactivation of neuronal chloride extrusion pumps; these processes result in inhibition of both axonal conduction and excitatory postsynaptic potentials, subsequently suppressing inhibitory postsynaptic potential formation and depolarizing neurons. ${ }^{16}$ The resulting total brain $\mathrm{NH}_{3}$ in $\mathrm{HE}$ may not reach an order of magnitude sufficient to alter the postsynaptic potentials, until advanced stages, but likely contributing to asterixis. As glutamine is the main amino acid involved in $\mathrm{HE}$, glutaminase haplotypes have been found to be associated with overt $\mathrm{HE}$, as well as increased mitochondrial $\mathrm{NH}_{3}$ resulting in reactive nitrogen and oxygen species worsening brain edema. ${ }^{17-19}$

Furthermore, as overt HE has been associated with elevated $\mathrm{NH}_{3}$ levels, recent studies suggest that various interactions with gut microbiota can play a crucial role in $\mathrm{HE}$. A proposed mechanism of dysbiosis in cirrhotics relates to bile acids. ${ }^{20}$ As commensal bacteria receive energy from primary bile acids that transition to secondary bile acids (i.e. cholic acid $\rightarrow$ deoxycholic acid), destabilization of bacterial membranes, along with increased intestinal permeability, results in an antimicrobial effect. ${ }^{21}$ As cirrhosis progresses to a decompensated state, the microbiome enters a dysbiosis state, ${ }^{22}$ leading to greater inflammation and cholestasis, in turn suppressing bile acid production in the liver and resulting in an overgrowth of pathogenic bacteria as the intestinal bile acids are decreased. Following the decrease in the ratio of secondary bile acids to primary bile acids, as seen in cirrhotics, the composition of gut microbiota becomes altered, resulting in a reduction in commensal bacteria. ${ }^{23}$ Cirrhotic patients have also been shown to have an increased Bacteroides/Firmicutes ratio, with changes in other pathogenic bacteria as well, including an increase in Enterobacteriaceae and a correlated reduction in the commensal bacterium Lachnospiraceae. ${ }^{24}$ The central role of gut microbiota in the pathogenesis of HE was recently demonstrated in a mouse model of cirrhosis (and compared to conventionally raised cirrhotic mice), wherein, under germ-free conditions, no brain inflammation or hyperammonemia was found to occur. ${ }^{25}$

Alternatively, proton pump inhibitors (PPIs) may contribute to the dysbiosis that is seen in patients with cirrhosis. The decrease in stomach acidity facilitates intestinal bacterial overgrowth and increases the risk of translocation of gut bacteria. ${ }^{26}$ This finding led to concerns that PPI may be a risk factor for the development of HE. Dam and colleagues ${ }^{26}$ used data from patients with ascites and cirrhosis who were enrolled in clinical trials evaluating the efficacy of satavaptan to examine whether PPIs increased the risk of first-time HE. The cumulative 1-year risk for the development of HE was $31 \%$ for those who used PPIs at baseline compared to $25 \%$ for those who did not. Similar results were reported from a case-control study conducted in Taiwan, which found that, in patients with cirrhosis and an occurrence of $\mathrm{HE}, 38 \%$ $(n=445)$ had a history of PPI use before HE occurrence. ${ }^{27}$

\section{Diagnosis}

HE tends to be a clinical diagnosis; however, covert HE can be more challenging (i.e. findings are normal in clinical examination) to diagnose than overt HE. Patients with covert HE will exhibit subtle signs and symptoms, like sleep-wake cycle disturbances; therefore, neuropsychological and psychometric tests are used to aid in the diagnosis. ${ }^{3,4,6,28}$ These psychometric tests are usually performed by experienced providers and may not be widely available. ${ }^{28,29}$ Furthermore, they are time-consuming and, as a result, are not widely used in clinical practice. ${ }^{3,4,6,29}$ However, the STROOP smartphone application is a short, valid, and reliable tool (c-statistic of 0.84 ) for screening covert HE patients. ${ }^{30}$

Alternatively, patients with overt HE demonstrating WHC stage 2 or 3, will exhibit mental or behavioral changes, such as confusion, disorientation, bizarre behavior and personality changes (Table 1). These patients can exhibit astrexis, tremors and/or ataxia, in addition to hyperreflexia and hypertonia. ${ }^{3}$ Patients with WHC stage $4 \mathrm{HE}$ are comatose and their exam will show diminished or absent deep tendon reflexes. The diagnosis of HE in these patients is often made based on clinical grounds after exclusion of other factors that may cause encephalopathy. 6,29,31

Elevated fasting $\mathrm{NH}_{3}$ levels in patients without clinical signs of $\mathrm{HE}$ was shown to correlate with the probability of occurrence of $\mathrm{HE}$ episodes. Patients that had $\mathrm{NH}_{3}$ values 1.5 times higher than the upper limit of normal (ULN) had a higher probability of developing an HE episode, as compared to patients with $\mathrm{NH}_{3}$ levels below 1.5 ULN (0.44 vs 0.22 , $p=0.003)$. Similarly, the annualized rates of HE-related hospitalizations and $\mathrm{HE}$ episodes were higher in patients with $\mathrm{NH}_{3}$ levels $>1.5$ ULN at baseline compared with those with normal $\mathrm{NH}_{3}$ or $>1$ ULN. ${ }^{32}$

Measurement of $\mathrm{NH}_{3}$ levels is not needed and does not add any diagnostic, staging or prognostic value in $\mathrm{HE}$ patients with 
Elwir S. et al: Hepatic encephalopathy

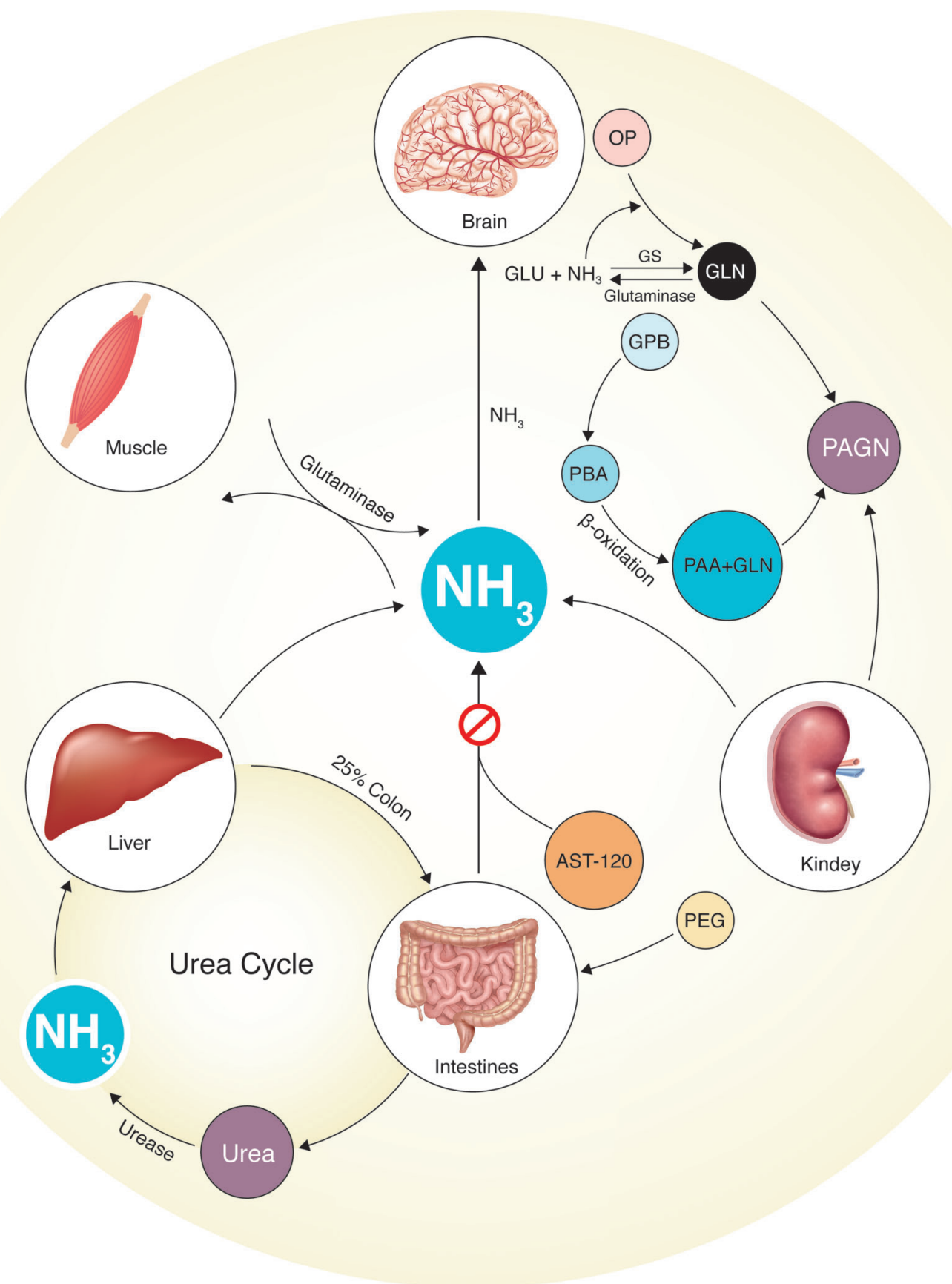

Fig. 1. Multiorgan ammonia pathways with specific ammonia-lowering medications used in cirrhosis. Circulating concentrations of ammonia $\left(\mathrm{NH}_{3}\right)$ are shown with multiorgan involvement in the production of $\mathrm{NH}_{3}$, ultimately resulting in $\mathrm{NH}_{3}$ crossing the blood-brain barrier, contributing to astrocyte swelling and hepatic encephalopathy, as decreased urea cycle capability and reduced liver glutamine synthetase activity is present in cirrhosis. The alternative pathway is also shown at the top, where $\mathrm{NH}_{3}$ binds with glutamate (GLU) forming glutamine (GLN) after enzymatic processing using glutamine synthetase (GS). Both ornithine phenylacetate (OP) and glycerol phenylbutyrate (GPB) are ammonia-lowering medications; they combine GLN and phenylacetate (PAA) to form phenylacetylglutamine (PAGN), which is excreted in the urine. AST-120 is a carbon microsphere adsorbent that binds $\mathrm{NH}_{3}$ in the gut, thus lowering circulating ammonia levels. Polyethylene glycol (PEG) is a cathartic, which causes rapid clearance of gut bacterial synthesizing ammonia to be excreted into the feces. About one-quarter of urea-derived byproducts from the urea cycle is shunted to the colon (not shown; remaining three-fourths of urea excreted in the kidneys), where urease-producing bacterial organisms produce ammonia that enters the portal circulation. Skeletal muscle also contributes to the regulation of $\mathrm{NH}_{3}$ as depicted. Not shown in the figure is the presence of $\mathrm{GS}$ and glutaminase in each organ, contributing to $\mathrm{NH}_{3}$ homeostasis. Adapted from publication by Thieme publications. ${ }^{76}$ Abbreviation: PBA, Phenylbutyric acid.

chronic liver disease. ${ }^{4}$ Furthermore, a previous study evaluated 59 cirrhotic patients and correlated $\mathrm{NH}_{3}$ levels using the WHC and critical flicker frequency (CFF) test, and found that up to $41 \%$ and $49 \%$ of patients were misdiagnosed if $\mathrm{NH}_{3}$ levels were used solely to diagnose $\mathrm{HE}$, as compared to using the WHC and CFF. ${ }^{33}$ The authors concluded that $\mathrm{NH}_{3}$ levels 
should not be used as a screening test and do not reliably detect $\mathrm{HE}$ in the emergency department, as frequent misinterpretations can arise when using $\mathrm{NH}_{3}$ blood levels in cirrhotic patients. ${ }^{33}$ Therefore, measurement of $\mathrm{NH}_{3}$ is not required for the diagnosis and management of these patients; however, if $\mathrm{NH}_{3}$ levels are normal in a patient with chronic liver disease and altered mental status (AMS), then a search for other factors that may cause the change in mental status should be sought. ${ }^{3,4}$

Evaluation of a patient with cirrhosis or portosystemic shunting and signs of overt HE starts with a search for alternative causes of AMS (such as stroke, hypercarbia, etc) and assessment of precipitating factors, correcting them if present (Table 2). 4,31,34 The evaluation includes obtaining history and performing a physical examination to investigate the presence of symptoms of gastrointestinal bleeding, constipation and infections that may have precipitated the HE. Labs are obtained to evaluate for electrolyte abnormalities, renal insufficiency and elevated white blood cell count. A full infectious work-up (including blood cultures, urine analysis and culture), chest X-ray and a diagnostic paracentesis should be undertaken as infections are a common precipitant for HE (Fig. 2). Commonly, patients will undergo cross-sectional imaging of the brain on admission.

One study showed that more than two-thirds of patients admitted with AMS in the setting of cirrhosis underwent brain imaging, but structural brain abnormalities were only found in patients who had focal neurological findings on physical examination. ${ }^{35}$ Another study by Donovan and colleagues ${ }^{36}$ evaluated the diagnostic yield of computed tomography (CT) scan in detecting intracranial hemorrhage in patients with cirrhosis and AMS; only $3 \%$ of the scans showed evidence of intracranial hemorrhage. The number needed to scan (NNS) for each positive result from CT varied by indication: focal neurologic deficits, NNS $=9$; fall/trauma, NNS $=20$; and AMS, NNS $=293 .{ }^{36}$

Assessment for medication noncompliance is a very important part of the evaluation. Data has shown that lack of adherence to a lactulose regimen was responsible for up to $38 \%$ of admissions with $\mathrm{HE} .{ }^{37}$ Lack of adherence is most commonly due to gastrointestinal side effects, such as bloating, abdominal pain and diarrhea, leading to inability to titrate bowel movements regularly. ${ }^{37}$ Up to $80 \%$ of patients may have a precipitant. ${ }^{4,34}$ When a precipitant is found, management of the precipitant, along with concomitant lactulose therapy (see below), is recommended. ${ }^{4,34}$ Large portosystemic shunts are found in up to $71 \%$ of patients with intractable $\mathrm{HE}^{38}$ and portosystemic shunts are a target for embolization in patients with intractable HE (see below).

\section{Table 2. Common precipitants of $\mathrm{HE}$}

\section{Gastrointestinal bleeding}

Infection

Use of centrally acting medications

Medication (lactulose and rifaximin) non-compliance

Renal failure and electrolyte abnormalities

Spontaneous portosystemic shunt

Constipation

\section{Treatment}

The two main forms of medical therapy for HE are nonabsorbale disaccharides (i.e. lacitol and lactulose) and nonabsorbable antibiotics (i.e. rifaximin). The hyperammonemia and inflammation that occurs due to urea breakdown in cirrhotics have led to development of HE treatments that target gut bacteria. ${ }^{39}$ Lactulose (B-galactosidofructose) and lactitol (B-galactosidosorbitol) are nonabsorbable disaccharides that are metabolized by the colonic microbiota to yield short chain organic acids. ${ }^{5}$ The resultant acidic environment is thought to prevent the growth of $\mathrm{NH}_{3}$-producing bacteria and promote the growth of beneficial microorganisms. The acidic environment also results in the change of $\mathrm{NH}_{3}$ to ammonium $\left(\mathrm{NH}_{4}{ }^{+}\right)$, which is not absorbed and decreases the ammonia load. Furthermore, the laxative effect results in removal of nitrogen-containing substances from the lumen of the gastrointestinal tract. ${ }^{5}$ Lactulose is administered orally or rectally and titrated to a goal of two to four bowel movements daily. ${ }^{4,15}$

A previous study demonstrated that $22 \%$ of 1 -month readmissions were preventable if the proper use of maintenance lactulose treatment and education was given to patients at discharge regarding the correct number of daily bowel movements that need to be achieved. ${ }^{40} \mathrm{~A}$ Cochrane review carried out in 2016 showed that, compared with placebo/no intervention, the nonabsorbable disaccharides were associated with overall beneficial effects on $\mathrm{HE}$ (relative risk: $0.58,95 \%$ confidence interval: 0.50 to 0.69$).{ }^{41}$ At present, lactulose is the first-line therapy for HE (although not approved by the US Food and Drug Administration for use in $\mathrm{HE}$ ) while treating the underlying precipitant of $\mathrm{HE}^{4}$ Alternatively, antibiotics work by altering the intestinal microbiota composition, and result in decreased production of ammonia. ${ }^{42}$ Rifaximin is the most commonly used nonabsorbable antibiotics and is typically applied as additive therapy to lactulose. Although prior research has evaluated the use of neomycin for overt $\mathrm{HE}_{1}{ }^{43}$ the side effect profile of ototoxicity and nephrotoxicity limits its current use. Other antibiotics such as metronidazole and vancomycin have also been studied, but their long-term use is limited due to potential side effects. $3,4,42$

In 2010, a multicenter study randomized patients who recovered from an episode of overt HE following treatment with rifaximin or placebo. Both groups were continued on lactulose and followed for a 6 -month period. A breakthrough episode of HE occurred in $22 \%(31 / 140)$ of patients in the rifaximin group, as compared with $46 \%(73 / 159)$ of patients in the placebo group, resulting in a number needed to treat of 4 patients to prevent one episode of overt HE. Furthermore, a decrease in hospitalization rates were seen in the rifaximin group (14\% (19/140), as compared with $23 \%(36 / 159)$ of patients in the placebo group), giving a hazard ratio of 0.50 (95\% confidence interval: 0.29 to $0.87 ; p=0.01$ ) and resulting in a number needed to treat of 9 patients to prevent one hospitalization. ${ }^{42}$

An open label study that expanded on the safety and efficacy of long-term (more than 24 months) rifaximin use demonstrated no increase in the rate of infections, including with Clostridium difficile, or in development of bacterial antibiotic resistance. Furthermore, rates of hospitalizations with long-term rifaximin administration remained low; the HE-related hospitalization rate, normalized for exposure (0.21 events per person-year of exposure; all-rifaximin 
Elwir S. et al: Hepatic encephalopathy
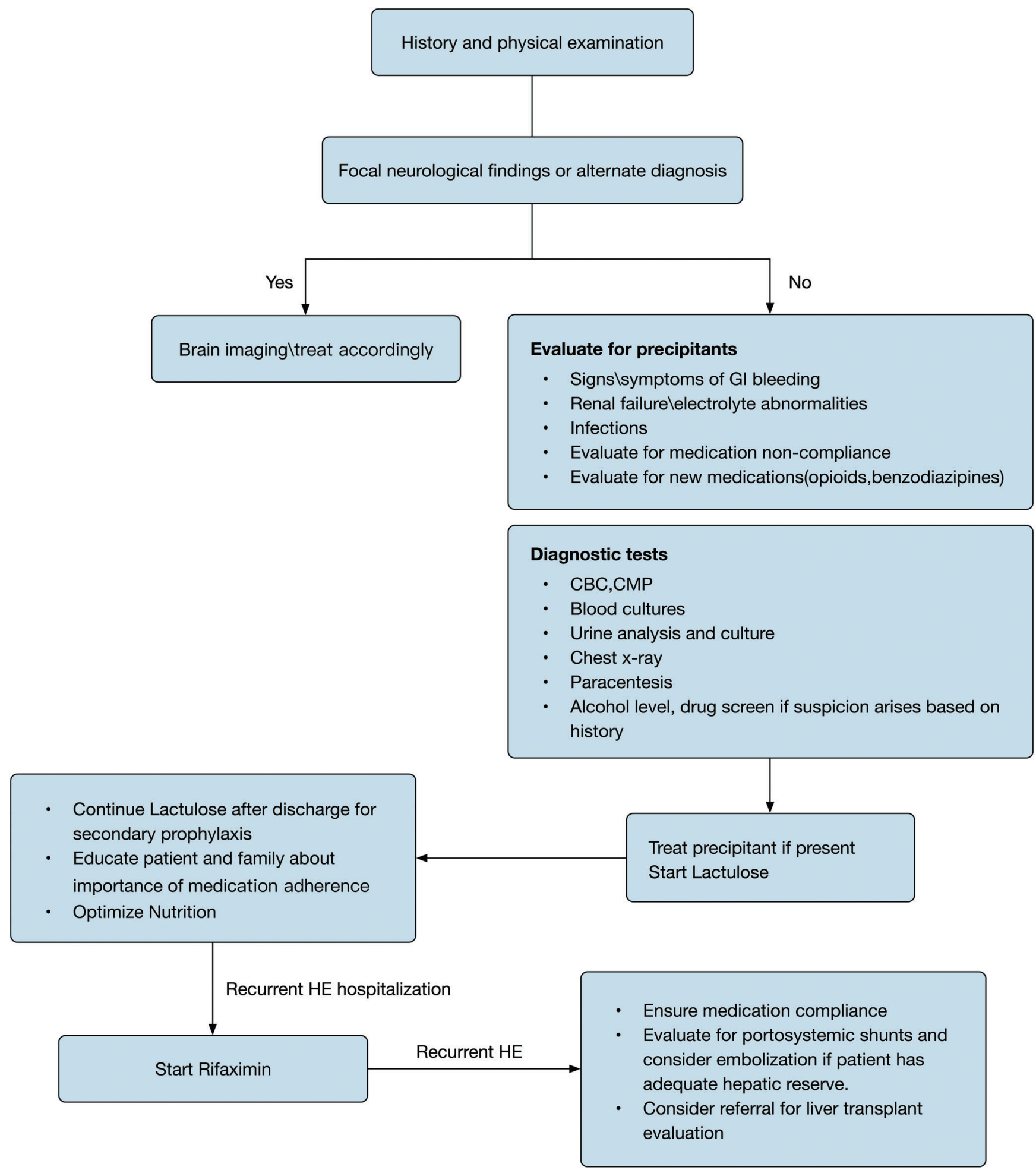

Fig. 2. Approach for addressing a patient with suspected HE.

population), was similar to that of the rifaximin group in the original randomized controlled trial (RCT) (0.30) and lower than that for the placebo group (0.72). ${ }^{44}$ Despite these data, only $60 \%$ of patients with a diagnosis of overt HE are on rifaximin, likely due to the high cost of this medication and lack of adequate insurance coverage. ${ }^{45}$ Data from Europe has shown that treatment with rifaximin can be cost effective through the related decrease in hospitalizations for $\mathrm{HE} .46,47$ 
Although cost of obtaining the medication for outpatient therapy is crucial, off-label use of rifaximin for overt HE was studied and 120 cirrhotic patients who were randomized to lactulose alone or lactulose and rifaximin treatment groups during hospitalization. Seventy-six percent of the patients in the combination group had complete reversal of $\mathrm{HE}$, as compared to $50.8 \%$ in the lactulose alone group $(p<0.004)$. Patients in the lactulose plus rifaximin group had shorter hospital stay $(5.8 \pm 3.4$ vs $8.2 \pm 4.6$ days, $p=0.001)$. There was also a significant decrease in mortality after treatment with lactulose plus rifaximin compared to lactulose and placebo $(23.8 \%$ vs $49.1 \%, p<0.05) .{ }^{48}$ Although more deaths were seen in lactulose group, no differences in mortality were associated with gastrointestinal bleeding or HRS between groups.

The current guidelines recommend addition of rifaximin to lactulose for the prevention of HE recurrence after the second episode of HE. ${ }^{4}$ Rifaximin is not recommended, however, as a stand-alone therapy at this point in time; however, further research is needed. ${ }^{4}$ Various studies have compared the effects of rifaximin as compared to other antibiotics and nonabsorbable disaccharides in the resolution of overt HE. A meta-analysis of these trials, reported in 2014, showed that rifaximin treatment was more likely to fully resolve an episode of overt $\mathrm{HE}$ (relative risk: 1.34, 95\% confidence interval: 1.11 to 1.62$)^{49}$ and had a beneficial effect on secondary prevention of HE (relative risk: 1.32; $95 \%$ confidence interval: 1.06 to 1.65$) .49$

Another issue that is often forgotten is the role of nutrition in the management of HE. Malnutrition is common in patients with cirrhosis and is associated with increased risk of sarcopenia and worsened survival. , $50-52$ Muscle tissue plays an important role in nitrogen metabolism and its loss is associated with increased risk of HE. Malnutrition is multifactorial in patients with cirrhosis with anorexia, early satiety, ascites, frequent paracentesis and AMS, and frequent hospitalizations lead to decreased protein intake and contribute to malnutrition as well. ${ }^{53}$ The International Society for Hepatic Encephalopathy and Nitrogen Metabolism released a consensus paper in 2013 outlining recommendations for nutritional management in patients with cirrhosis. ${ }^{52}$ The optimal daily energy intake should be $35-40 \mathrm{kcal} / \mathrm{kg}$ ideal body weight. The optimal daily protein intake should be $1.2-1.5 \mathrm{~g} / \mathrm{kg}$ ideal body weight. The publication suggested that small meals, evenly distributed throughout the day, and a late-night snack of complex carbohydrates will minimize protein utilization. ${ }^{52}$ In a study by Plank and colleagues, ${ }^{51}$ provision of night time supplementation was associated with an increase in total body protein stores, as compared to day time feedings. Compliance was an issue in that study, with some patients having difficulty in consuming the full volume of the supplements or taking it all at night time. In those patients, branched chain fatty acids can be used (see below). ${ }^{4,52}$

\section{Other treatment regimens}

\section{Polyethylene glycol (PEG)}

A recent trial comparing PEG and lactulose found that PEG led to more rapid overt $\mathrm{HE}$ resolution within 24 hours compared to the standard-of-care lactulose, suggesting that PEG may be superior to standard lactulose therapy in patients with cirrhosis hospitalized for acute $\mathrm{HE}^{54}$ (Fig. 1). Although nasogastric tube placement was used to administer PEG in patients with severe overt $\mathrm{HE}$, difficulty in administering a large volume of PEG in patients with AMS may limit the use of this approach if nasogastric tube placement cannot be achieved.

\section{Glycerol phenylbutyrate (GPB)}

GPB is a colorless (to pale yellow), nearly tasteless, sodiumfree, odorless liquid, which removes nitrogen in the form of urinary phenylacetylglutamine (PAGN), allowing for excretion of $\mathrm{NH}_{3}$ (Fig. 1). As GPB has been shown to be well tolerated, ${ }^{55}$ a multicenter, RCT phase II trial was conducted. ${ }^{56}$ Out of 178 cirrhotic patients, 61\% (55/90) in the GPB arm ( $v s$ $76 \%(67 / 88)$ in the placebo arm) were included in the intention-to-treat analysis. Patients in the GPB group experienced less HE events than those in the placebo group (the primary endpoint; $21 \%$ vs $36 \%, p=0.02$ ). In addition, time to first event (hazard ratio: $0.56, p<0.05$ ), fewer HE hospitalizations (13 vs 25, $p=0.06$ ) and total events (35 vs 57, $p=0.04$ ) favored the GPB arm over the placebo arm. Among patients not taking rifaximin at enrollment, GPB had even more prominent effects, reducing the proportion of patients with an HE event ( $10 \%$ vs $32 \%, p<0.01)$, total events ( 7 vs 31, $p<0.01$ ), and time to first event (hazard ratio: $0.29 ; p<0.01$ ). Plasma $\mathrm{NH}_{3}$ levels were significantly lower in the GPB arm, while adverse events (about $75 \%$ in each group) did not differ between the two groups. Therefore, GPB decreased $\mathrm{NH}_{3}$ and was considered safe and capable of improving outcomes in cirrhotic patients with recurrent $\mathrm{HE}$. Future investigations are anticipated to further establish the role of GPB in cirrhotic patients with HE.

\section{Ornithine phenylacetate (OP)}

OP (OCR-002) combines ornithine and phenylacetate (PAA) in a crystalline salt and removes nitrogen in the form of urinary PAGN (Fig. 1). Its safety and tolerability was demonstrated in upper gastrointestinal cirrhotic patients without $\mathrm{HE}$, in a study which explored effects on blood ammonia and urine metabolites during a 5-day continuous infusion. ${ }^{57}$ As expected, OP infusion decreased plasma $\mathrm{NH}_{3}$ levels at every 24-hour time point up to 5 days, with plasma glutamine also being decreased and an increasing concentration of urinary PAGN throughout the 5 days. No serious adverse events were reported. Currently, we are awaiting the results of a multicenter randomized, double-blind, placebo controlled phase $2 \mathrm{~b}$ trial evaluating $\mathrm{OP}$ in overt $\mathrm{HE}$, with results expected in mid-2017.

\section{Probiotics}

Multiple studies have looked at the use of probiotics for the treatment of HE. ${ }^{3,4}$ One study, in which 160 patients with HE were randomly assigned to receive probiotics 3 times daily or no probiotics, was conducted to assess the development of overt HE over a mean follow-up of 40 weeks \pm 10 weeks after receiving 3 months of probiotics. Of the 86 patients (42 with covert $\mathrm{HE}$ ) receiving probiotics, 7 subjects developed overt $\mathrm{HE}$, compared to 74 patients ( 33 with covert $\mathrm{HE}$ ) among the controls, of which 14 developed overt $\mathrm{HE}$ $(p<0.05)$, as determined after psychometric testing was performed. There were no differences in overall mortality between the two groups. This study concluded that, in patients without prior overt $\mathrm{HE}$, probiotics can be useful in 
preventing overt $\mathrm{HE} ;{ }^{58}$ however, longer follow-up studies are needed to assess probiotic efficacy.

Another study focused on secondary prophylaxis for HE cirrhotic patients. A total of 235 cirrhotic patients, who had recovered from overt $\mathrm{HE}$, were randomized to receive either lactulose ( $30 \mathrm{~mL} 3$ times a day; $n=80$ ), probiotics ( 3 capsules daily, containing 112.5 billion viable lyophilized bacteria per capsule; $n=77)$ or placebo $(n=78)$ and were followed for 1 year and tested for development of overt HE by psychometry and using the WHC. Using intention to treat analysis, 30/80 (38\%) receiving lactulose, 35/77 (45\%) receiving probiotics and $50 / 78(64 \%)$ receiving no therapy developed recurrent $\mathrm{HE},(p=0.003)$. There were significant differences seen in the cases of lactulose or probiotics given for secondary prophylaxis, as compared to placebo ( $p=0.001$ and $p=0.02$ ); however, there were no differences when direct comparisons were made between lactulose and probiotics respectively $(p=0.35) .^{59}$

A recent meta-analysis showed that compared to placebo, probiotic use was associated with improvement in covert $\mathrm{HE}$, decreased hospitalization and decreased progression to overt $\mathrm{HE}$ in patients with minimal (i.e. covert) HE. ${ }^{60}$ However, these effects were not observed when probiotic use was compared to lactulose. In addition, probiotics were not associated with any effects on mortality when compared to placebo and lactulose. ${ }^{60}$

\section{Branched chain amino acids (BCAAs)}

Patients with cirrhosis have an imbalance between aromatic amino acids and BCAAs. ${ }^{31}$ It has been suggested that this imbalance contributes to the development of HE. ${ }^{31} \mathrm{~A}$ Cochrane review of 16 RCTs comparing BCAA to placebo, no intervention, diet, neomycin or lactulose revealed that BCAAs had a beneficial effect on HE (relative risk: $0.73,95 \%$ confidence interval: 0.61 to 0.88$){ }^{61}$ In sensitivity analyses that excluded trials with a lactulose or neomycin control, BCAAs had a beneficial effect on HE (relative risk: 0.76 , 95\% confidence interval: 0.63 to 0.92); however, there was no difference between BCAAs and lactulose or neomycin (relative risk: $0.66,95 \%$ confidence interval: 0.34 to 1.30$){ }^{61}$ The most up to date recommendations suggest that oral BCAAs can be used as an alternative or additional agent to treat patients nonresponsive to conventional therapy. ${ }^{4}$

\section{Zinc}

Some of the urea cycle enzymes are zinc-dependent. ${ }^{5}$ Zinc deficiency is prevalent in patients with cirrhosis. ${ }^{34}$ Multiple trials have evaluated the use of zinc in the treatment of $\mathrm{HE}$, yielding variable results. A meta-analysis of four RCTs showed that zinc supplementation improved the results of some psychometric tests but not of HE recurrence. ${ }^{31}$ In the setting of zinc deficiency or confirmed losses, zinc supplementation can be considered when treating HE. ${ }^{4}$

\section{L-Ornithine-L-Aspartate (LOLA)}

LOLA lowers plasma ammonia concentrations by enhancing the metabolism of ammonia to glutamine. ${ }^{5,34}$ The efficacy of LOLA was evaluated in a meta-analysis by Bai and colleagues $^{62}$ that showed that when comparing to a placebo/ no-intervention control, LOLA was significantly more effective in the improvement of HE (relative risk: $1.49,95 \%$ confidence interval: 1.10 to 2.01). Two studies that compared LOLA and lactulose showed similar efficacy in the treatment of $\mathrm{HE}$ (relative risk: $0.88,95 \%$ confidence interval: 0.57 to 1.35$).{ }^{62}$ Intravenous LOLA can be used as an alternative or additional agent to treat patients nonresponsive to conventional therapy; ${ }^{4}$ however, LOLA is not currently available in the US.

\section{Portosystemic shunt embolization}

Embolization of the portosystemic shunts is thought to offer a therapeutic target in patients with intractable HE. ${ }^{4}$ The concern is that, with embolization, blood is shunted to the portal system and as a result causes an increase in portal hypertension and portal hypertensive complications. Various centers have reported on their outcomes and success rates with these techniques, with varying results. In a study reported by Lynn and colleagues, ${ }^{63} 20$ patients underwent embolization of large portosystemic shunts for medically refractory $\mathrm{HE}$. The mean preprocedural MELD score was $13.1 \pm 3.4$. Prior to embolization, $14(70 \%)$ patients had overt, preexisting portal hypertension. The procedure was technically successful in all patients, with immediate significant improvement in symptoms occurring within 1 week after the procedure. Long-term follow-up information was available for $12 / 20$ patients, with no HE reported hospitalizations within 12 months of the procedure for $8 / 12$ patients. Within the follow-up period, 7 patients presented evidence of worsening portal hypertension; one developed small nonbleeding varices on endoscopy and six had new or worsening ascites. ${ }^{63}$

Similar results were seen in the multicenter European study, in which 37 patients with a mean MELD score of 13.2 \pm 0.9 underwent embolization of portosystemic shunts. On a short-term basis (i.e. within 100 days after embolization), $59.4 \%(22 / 37)$ of the patients were free of $\mathrm{HE}$ ( $v s$ before embolization, $p<0.001$ ), of which 18 (or $48.6 \%$ of patients overall) remained $\mathrm{HE}$-free over a mean period of follow-up of $697 \pm 157$ days ( $v s$ before embolization, $p<0.001$ ). In this study, there was no significant aggravation of portal hypertension during follow-up. ${ }^{64}$ Naeshiro et al. ${ }^{65}$ reported resolution of $\mathrm{HE}$ in $13 / 14$ patients who underwent embolization, and aggravation in varices in $29 \%$ of patients (4/14). Another series reported by Zidi et al. ${ }^{66}$ on 7 patients with cirrhosis (mean Child-Pugh score of B9) and intractable HE resulted in sustained improvement in only 1 patient. Four patients died during the follow-up period, two from sepsis and coma and two from variceal bleeding. A pre-embolization MELD score of 11 or more was found to be associated with higher chances of recurrent $\mathrm{HE}$ after embolization in the study by Laleman et al. ${ }^{64}$ Singh et al. ${ }^{67}$ proposed a pre-embolization MELD score of 15 as a cut-off to identify potential candidates for embolization. ${ }^{64}$

\section{Post-TIPS}

HE occurs after TIPS placement in $10-50 \%$ of patients. ${ }^{7}$ An RCT evaluated the efficacy of rifaximin, lactitol or no treatment on the incidence of HE after TIPS placement. ${ }^{68}$ The incidence of HE was found to be similar for the three groups. ${ }^{68}$ The current guidelines do not recommend routine prophylaxis for prevention of post-TIPS HE. ${ }^{4}$ 
Elwir S. et al: Hepatic encephalopathy

\section{Extracorporeal devices}

Therapeutic alternatives, like extracorporeal devices that remove $\mathrm{NH}_{3}$ from the body have been used for cirrhotic patients with refractory $\mathrm{HE}$ and acute liver failure with $\mathrm{HE}$. The Molecular Adsorbent Recirculating System (MARS; Baxter Intl., Deerfield, IL, US) represents the classic device, which was granted Food and Drug Administration approval in January 2013 for the treatment of $\mathrm{HE}$, and is used in nearly 50 countries worldwide, although mostly for research purposes as it is considered a costly treatment. However, a multicenter RCT in patients with severe HE not responding to standard medical therapy (SMT) was conducted using the MARS system. ${ }^{69}$ In that study, MARS was used for 6 hours daily for 5 days or until the patient had at least a 2-grade improvement in HE when compared to SMT. A total of 70 patients (median age: 53; median MELD: 32 ), 66\% of who were male, having severe overt HE (grade 3, 56\%; grade $4,44 \%)$, were randomized to MARS plus SMT $(n=39)$ or SMT alone $(n=31)$. Higher improvement of HE was seen in the MARS plus SMT group (34\%) compared to the SMT group $(19 \%, p=0.044)$. Overall, HE resolution was reached at a more rapid rate than with the SMT group $(p=0.045)$, and was tolerated well; however, there was no benefit in regards to mortality between the two groups.

\section{Liver Transplantation}

Liver transplantation represents the ultimate treatment for decompensated cirrhosis. Although overt HE significantly improves and reverses after transplant, some patients continue to show signs of cognitive impairment. In a study by Campagna et al., ${ }^{70}$ patients with cirrhosis (with or without history of $\mathrm{HE}$ ) had psychometric evaluation for $\mathrm{HE}$ and electroencephalograms (EEGs) both before and after transplant. Patients with a history of overt HE showed greater improvements after liver transplantation than patients with a negative history, but their global cognitive function remained slightly worse; in contrast, EEGs normalized in both groups. Another study evaluated patients with minimal HE who had undergone liver transplantation with a battery of neuropsychological tests. Results showed that the visuomotor deficits subside or disappear only in some of the patients after transplantation, while a significant number of patients show no improvement in the visuomotor and visuoconstructive functions, as measured by the neuropsychological tests. ${ }^{71}$ Sotil et al. ${ }^{72}$ evaluated the impact of pre-transplant overt HE on posttransplant cognitive function and noted that patients with pre-transplant HE scored below the control group when their cognitive function was assessed by two neuropsychological battery tests. This difference, however, did not affect the quality of life of these patients. Another study by GarciaMartinez et al. ${ }^{73}$ showed persistence of mild cognitive impairment in only $13 \%$ of patients with HE before transplant. Brain volumes were also smaller in the patients with HE before transplant. ${ }^{73}$ Persistent portosystemic shunts can result in post-transplant $\mathrm{HE}$, and embolization of these shunts is reported to result in complete resolution of symptoms. ${ }^{74,75}$

\section{Conclusion}

HE is a common but reversible neuropsychiatric problem in patients with cirrhosis and portosystemic shunting, and is associated with significant morbidity and health care costs as well as increased mortality. The approach to diagnosis and treatment centers on ruling out and correcting/limiting any underlying precipitating factors, while treating with nonabsorbable disaccharides (i.e. lactulose) with or without nonabsorbable antibiotics (rifaximin), in conjunction with educating family members on how titrating therapy is crucial to preventing readmissions. Other therapies, including probiotics, zinc and BCAAs, can be considered for patients who fail to respond to initial measures. Furthermore, splenorenal shunt embolization can be considered for recurrent HE patients with low MELD scores, if standard therapy has been exhausted. Studies of potential therapies with OP are underway; however, we are awaiting the results of that particular phase $2 \mathrm{~b}$ multicenter RCT, which are due out in 2017, before determinations can be made on its use in $\mathrm{HE}$.

\section{Conflict of interest}

Rahimi R.S. received research support from Ocera Therapeutics. The others has no conflict of interest related to this publication.

\section{Author contributions}

Data collection and drafting and revising of the manuscript (SE, RSR).

\section{References}

[1] Xu J, Murphy SL, Kochanek KD, Bastian BA. Deaths: final data for 2013. Natl Vital Stat Rep 2016;64:1-119.

[2] Bajaj JS, Wade JB, Gibson DP, Heuman DM, Thacker LR, Sterling RK, et al. The multi-dimensional burden of cirrhosis and hepatic encephalopathy on patients and caregivers. Am J Gastroenterol 2011;106:1646-1653. doi: 10.1038/ajg.2011.157.

[3] Patidar KR, Bajaj JS. Covert and overt hepatic encephalopathy: diagnosis and management. Clin Gastroenterol Hepatol 2015;13:2048-2061. doi: 10.1016/j.cgh.2015.06.039.

[4] Vilstrup H, Amodio P, Bajaj J, Cordoba J, Ferenci P, Mullen KD, et al. Hepatic encephalopathy in chronic liver disease: 2014 Practice Guideline by the American Association for the Study of Liver Diseases and the European Association for the Study of the Liver. Hepatology 2014;60:715-735. doi: 10.1002/hep. 27210.

[5] Gerber T, Schomerus H. Hepatic encephalopathy in liver cirrhosis: pathogenesis, diagnosis and management. Drugs 2000;60:1353-1370. doi: 10 . 2165/00003495-200060060-00008

[6] Ferenci P, Lockwood A, Mullen K, Tarter R, Weissenborn K, Blei AT. Hepatic encephalopathy-definition, nomenclature, diagnosis, and quantification: final report of the working party at the 11th World Congresses of Gastroenterology, Vienna, 1998. Hepatology 2002;35:716-721. doi: 10.1053/ jhep.2002.31250.

[7] Poordad FF. Review article: the burden of hepatic encephalopathy. Aliment Pharmacol Ther 2007;25 Suppl 1:3-9. doi: 10.1111/j.1746-6342.2006. 03215.x.

[8] Stepanova M, Mishra A, Venkatesan C, Younossi ZM. In-hospital mortality and economic burden associated with hepatic encephalopathy in the United States from 2005 to 2009. Clin Gastroenterol Hepatol 2012;10:1034-1041. e1. doi: 10.1016/j.cgh.2012.05.016.

[9] Bajaj JS, Reddy KR, Tandon P, Wong F, Kamath PS, Garcia-Tsao G, et al. The 3-month readmission rate remains unacceptably high in a large North American cohort of patients with cirrhosis. Hepatology 2016;64:200-208. doi: $10.1002 /$ hep. 28414 .

[10] Bustamante J, Rimola A, Ventura PJ, Navasa M, Cirera I, Reggiardo V, et al. Prognostic significance of hepatic encephalopathy in patients with cirrhosis. J Hepatol 1999;30:890-895. doi: 10.1016/S0168-8278(99)80144-5.

[11] Stewart CA, Malinchoc M, Kim WR, Kamath PS. Hepatic encephalopathy as a predictor of survival in patients with end-stage liver disease. Liver Transpl 2007;13:1366-1371. doi: 10.1002/It.21129.

[12] Wong RJ, Gish RG, Ahmed A. Hepatic encephalopathy is associated with significantly increased mortality among patients awaiting liver transplantation. Liver Transpl 2014;20:1454-1461. doi: 10.1002/It.23981. 
[13] Wong RJ, Aguilar M, Gish RG, Cheung R, Ahmed A. The impact of pretransplant hepatic encephalopathy on survival following liver transplantation. Liver Transpl 2015;21:873-880. doi: 10.1002/It.24153.

[14] Tapper EB, Jiang ZG, Patwardhan VR. Refining the ammonia hypothesis: a physiology-driven approach to the treatment of hepatic encephalopathy. Mayo Clin Proc 2015;90:646-658. doi: 10.1016/j.mayocp.2015.03.003.

[15] Wijdicks EF. Hepatic encephalopathy. N Engl J Med 2016;375:1660-1670. doi: $10.1056 /$ NEJMra1600561.

[16] Szerb JC, Butterworth RF. Effect of ammonium ions on synaptic transmission in the mammalian central nervous system. Prog Neurobiol 1992;39: 135-153. doi: 10.1016/0301-0082(92)90008-3.

[17] Albrecht J, Norenberg MD. Glutamine: a Trojan horse in ammonia neurotoxicity. Hepatology 2006;44:788-794. doi: 10.1002/hep.21357.

[18] Romero-Gómez M, Jover M, Del Campo JA, Royo JL, Hoyas E, Galán JJ, et al. Variations in the promoter region of the glutaminase gene and the development of hepatic encephalopathy in patients with cirrhosis: a cohort study. Ann Intern Med 2010;153:281-288. doi: 10.7326/0003-4819-1535-201009070-00002.

[19] Jayakumar AR, Rama Rao KV, Schousboe A, Norenberg MD. Glutamineinduced free radical production in cultured astrocytes. Glia 2004;46: 296-301. doi: 10.1002/glia.20003.

[20] Bajaj JS, Heuman DM, Hylemon PB, Sanyal AJ, White MB, Monteith P, et al. Altered profile of human gut microbiome is associated with cirrhosis and its complications. J Hepatol 2014;60:940-947. doi: 10.1016/j.jhep.2013. 12.019.

[21] Karlsson F, Tremaroli V, Nielsen J, Bäckhed F. Assessing the human gut microbiota in metabolic diseases. Diabetes 2013;62:3341-3349. doi: 10. 2337/db13-0844.

[22] Shawcross D, Jalan R. The pathophysiologic basis of hepatic encephalopathy: central role for ammonia and inflammation. Cell Mol Life Sci 2005 ; 62:2295-2304. doi: 10.1007/s00018-005-5089-0.

[23] Kakiyama G, Pandak WM, Gillevet PM, Hylemon PB, Heuman DM, Daita K, et al. Modulation of the fecal bile acid profile by gut microbiota in cirrhosis. J Hepatol 2013;58:949-955. doi: 10.1016/j.jhep.2013.01.003.

[24] Liu Q, Duan ZP, Ha DK, Bengmark S, Kurtovic J, Riordan SM. Synbiotic modulation of gut flora: effect on minimal hepatic encephalopathy in patients with cirrhosis. Hepatology 2004;39:1441-1449. doi: 10.1002/hep.20194.

[25] Kang D], Betrapally NS, Ghosh SA, Sartor RB, Hylemon PB, Gillevet PM, et al. Gut microbiota drive the development of neuroinflammatory response in cirrhosis in mice. Hepatology 2016;64:1232-1248. doi: 10.1002/hep. 28696.

[26] Dam G, Vilstrup H, Watson H, Jepsen P. Proton pump inhibitors as a risk factor for hepatic encephalopathy and spontaneous bacterial peritonitis in patients with cirrhosis with ascites. Hepatology 2016;64:1265-1272. doi: 10.1002/hep. 28737.

[27] Tsai CF, Chen MH, Wang YP, Chu CJ, Huang YH, Lin HC, et al. Proton pump inhibitors increase risk for hepatic encephalopathy in patients with cirrhosis in a population study. Gastroenterology 2017;152:134-141. doi: 10.1053/j. gastro.2016.09.007

[28] Goldbecker A, Weissenborn K, Hamidi Shahrezaei G, Afshar K, Rümke S Barg-Hock $\mathrm{H}$, et al. Comparison of the most favoured methods for the diagnosis of hepatic encephalopathy in liver transplantation candidates. Gut 2013;62:1497-1504. doi: 10.1136/gutjnl-2012-303262.

[29] Elwir S, Hal H, Veith J, Schreibman I, Kadry Z, Riley T. Radiographical findings in patients with liver cirrhosis and hepatic encephalopathy. Gastroenterol Rep (Oxf) 2016;4:221-225. doi: 10.1093/gastro/gov049.

[30] Bajaj JS, Thacker LR, Heuman DM, Fuchs M, Sterling RK, Sanyal AJ, et al. The Stroop smartphone application is a short and valid method to screen for minimal hepatic encephalopathy. Hepatology 2013;58:1122-1132. doi: 10.1002/hep.26309.

[31] Jawaro T, Yang A, Dixit D, Bridgeman MB. Management of hepatic encephalopathy: a primer. Ann Pharmacother 2016;50:569-577. doi: 10 . $1177 / 1060028016645826$.

[32] Vierling JM, Mokhtarani M, Brown RS Jr, Mantry P, Rockey DC, Ghabril M, et al. Fasting blood ammonia predicts risk and frequency of hepatic encephalopathy episodes in patients with cirrhosis. Clin Gastroenterol Hepatol 2016;14:903-906.e1. doi: 10.1016/j.cgh.2015.11.018.

[33] Gundling F, Zelihic E, Seidl H, Haller B, Umgelter A, Schepp W, et al. How to diagnose hepatic encephalopathy in the emergency department. Ann Hepatol 2013;12:108-114.

[34] Leise MD, Poterucha J], Kamath PS, Kim WR. Management of hepatic encephalopathy in the hospital. Mayo Clin Proc 2014;89:241-253. doi: 10. 1016/j.mayocp.2013.11.009.

[35] Rahimi RS, Rockey DC. Overuse of head computed tomography in cirrhosis with altered mental status. Am J Med Sci 2016;351:459-466. doi: 10 1016/j.amjms.2016.02.022.

[36] Donovan LM, Kress WL, Strnad LC, Sarwar A, Patwardhan V, Piatkowski G, et al. Low likelihood of intracranial hemorrhage in patients with cirrhosis and altered mental status. Clin Gastroenterol Hepatol 2015;13:165-169. doi: $10.1016 /$ j.cgh.2014.05.022.
[37] Bajaj JS, Sanyal AJ, Bell D, Gilles H, Heuman DM. Predictors of the recurrence of hepatic encephalopathy in lactulose-treated patients. Aliment Pharmacol Ther 2010;31:1012-1017. doi: 10.1111/j.1365-2036.2010.04257.x.

[38] Riggio O, Efrati C, Catalano C, Pediconi F, Mecarelli O, Accornero N, et al. High prevalence of spontaneous portal-systemic shunts in persistent hepatic encephalopathy: a case-control study. Hepatology 2005;42:1158-1165. doi: $10.1002 /$ hep.20905.

[39] Riordan SM, Williams R. Gut flora and hepatic encephalopathy in patients with cirrhosis. N Engl J Med 2010;362:1140-1142. doi: 10.1056/ NEJMe1000850.

[40] Volk ML, Tocco RS, Bazick J, Rakoski MO, Lok AS. Hospital readmissions among patients with decompensated cirrhosis. Am J Gastroenterol 2012; 107:247-252. doi: 10.1038/ajg.2011.314

[41] Gluud LL, Vilstrup H, Morgan MY. Non-absorbable disaccharides versus placebo/no intervention and lactulose versus lactitol for the prevention and treatment of hepatic encephalopathy in people with cirrhosis. Cochrane Database Syst Rev 2016;4:CD003044. doi: 10.1002/14651858.CD003044. pub3.

[42] Bass NM, Mullen KD, Sanyal A, Poordad F, Neff G, Leevy CB, et al. Rifaximin treatment in hepatic encephalopathy. N Engl J Med 2010;362:1071-1081. doi: 10.1056/NEJMoa0907893.

[43] Strauss E, Tramote R, Silva EP, Caly WR, Honain NZ, Maffei RA, et al. Doubleblind randomized clinical trial comparing neomycin and placebo in the treatment of exogenous hepatic encephalopathy. Hepatogastroenterology 1992; 39:542-545.

[44] Mullen KD, Sanyal AJ, Bass NM, Poordad FF, Sheikh MY, Frederick RT, et al. Rifaximin is safe and well tolerated for long-term maintenance of remission from overt hepatic encephalopathy. Clin Gastroenterol Hepatol 2014;12: 1390-1397.e2. doi: 10.1016/j.cgh.2013.12.021.

[45] Landis CS, Ghabril M, Rustgi V, Di Bisceglie AM, Maliakkal B, Rockey DC, et al. Prospective multicenter observational study of overt hepatic encephalopathy. Dig Dis Sci 2016;61:1728-1734. doi: 10.1007/s10620-016-4031-7.

[46] Orr JG, Currie CJ, Berni E, Goel A, Moriarty KJ, Sinha A, et al. The impact on hospital resource utilisation of treatment of hepatic encephalopathy with rifaximin- $\alpha$. Liver Int 2016;36:1295-1303. doi: 10.1111/liv.13111.

[47] Kabeshova A, Ben Hariz S, Tsakeu E, Benamouzig R, Launois R. Costeffectiveness analysis of rifaximin- $\alpha$ administration for the reduction of episodes of overt hepatic encephalopathy in recurrence compared with standard treatment in France. Therap Adv Gastroenterol 2016;9:473-482. doi: $10.1177 / 1756283 \times 16644249$

[48] Sharma BC, Sharma P, Lunia MK, Srivastava S, Goyal R, Sarin SK. A randomized, double-blind, controlled trial comparing rifaximin plus lactulose with lactulose alone in treatment of overt hepatic encephalopathy. Am J Gastroenterol 2013;108:1458-1463. doi: 10.1038/ajg.2013.219.

[49] Kimer N, Krag A, Møller S, Bendtsen F, Gluud LL. Systematic review with meta-analysis: the effects of rifaximin in hepatic encephalopathy. Aliment Pharmacol Ther 2014;40:123-132. doi: 10.1111/apt.12803.

[50] Alberino F, Gatta A, Amodio P, Merkel C, Di Pascoli L, Boffo G, et al. Nutrition and survival in patients with liver cirrhosis. Nutrition $2001 ; 17: 445-450$. doi: 10.1016/S0899-9007(01)00521-4.

[51] Plank LD, Gane EJ, Peng S, Muthu C, Mathur S, Gillanders L, et al. Nocturnal nutritional supplementation improves total body protein status of patients with liver cirrhosis: a randomized 12-month trial. Hepatology 2008;48: 557-566. doi: 10.1002/hep.22367.

[52] Amodio P, Bemeur C, Butterworth R, Cordoba J, Kato A, Montagnese S, et al. The nutritional management of hepatic encephalopathy in patients with cirrhosis: International Society for Hepatic Encephalopathy and Nitrogen Metabolism Consensus. Hepatology 2013;58:325-336. doi: 10.1002/hep. 26370.

[53] Chadalavada R, Sappati Biyyani RS, Maxwell J, Mullen K. Nutrition in hepatic encephalopathy. Nutr Clin Pract 2010;25:257-264. doi: 10.1177/ 0884533610368712

[54] Rahimi RS, Singal AG, Cuthbert JA, Rockey DC. Lactulose vs polyethylene glycol 3350-electrolyte solution for treatment of overt hepatic encephalopathy: the HELP randomized clinical trial. JAMA Intern Med 2014;174: 1727-1733. doi: 10.1001/jamainternmed.2014.4746.

[55] Ghabril M, Zupanets IA, Vierling J, Mantry P, Rockey D, Wolf D, et al. Glycerol phenylbutyrate in patients with cirrhosis and episodic hepatic encephalopathy: a pilot study of safety and effect on venous ammonia concentration. Clin Pharmacol Drug Dev 2013;2:278-284. doi: 10.1002/cpdd.18.

[56] Rockey DC, Vierling JM, Mantry P, Ghabril M, Brown RS Jr, Alexeeva O, et al. Randomized, double-blind, controlled study of glycerol phenylbutyrate in hepatic encephalopathy. Hepatology 2014;59:1073-1083. doi: 10.1002/ hep. 26611.

[57] Ventura-Cots M, Arranz JA, Simón-Talero M, Torrens M, Blanco A, Riudor E, et al. Safety of ornithine phenylacetate in cirrhotic decompensated patients: an open-label, dose-escalating, single-cohort study. J Clin Gastroenterol 2013;47:881-887. doi: 10.1097/MCG.0b013e318299c789.

[58] Lunia MK, Sharma BC, Sharma P, Sachdeva S, Srivastava S. Probiotics prevent hepatic encephalopathy in patients with cirrhosis: a randomized 
controlled trial. Clin Gastroenterol Hepatol 2014;12:1003-1008.e1. doi: 10 1016/j.cgh.2013.11.006.

[59] Agrawal A, Sharma BC, Sharma P, Sarin SK. Secondary prophylaxis of hepatic encephalopathy in cirrhosis: an open-label, randomized controlled trial of lactulose, probiotics, and no therapy. Am J Gastroenterol 2012;107: 1043-1050. doi: 10.1038/ajg.2012.113.

[60] Saab S, Suraweera D, Au J, Saab EG, Alper TS, Tong MJ. Probiotics are helpful in hepatic encephalopathy: a meta-analysis of randomized trials. Liver Int 2016;36:986-993. doi: 10.1111/liv.13005.

[61] Gluud LL, Dam G, Les I, Córdoba J, Marchesini G, Borre M, et al. Branchedchain amino acids for people with hepatic encephalopathy. Cochrane Database Syst Rev 2015;(9):CD001939. doi: 10.1002/14651858.CD001939. pub3.

[62] Bai M, Yang Z, Qi X, Fan D, Han G. I-ornithine-l-aspartate for hepatic encephalopathy in patients with cirrhosis: a meta-analysis of randomized controlled trials. J Gastroenterol Hepatol 2013;28:783-792. doi: 10.1111/ jgh.12142.

[63] Lynn AM, Singh S, Congly SE, Khemani D, Johnson DH, Wiesner RH, et al. Embolization of portosystemic shunts for treatment of medically refractory hepatic encephalopathy. Liver Transpl 2016;22:723-731. doi: 10.1002/lt. 24440.

[64] Laleman W, Simon-Talero M, Maleux G, Perez M, Ameloot K, Soriano G, et al. Embolization of large spontaneous portosystemic shunts for refractory hepatic encephalopathy: a multicenter survey on safety and efficacy. Hepatology 2013;57:2448-2457. doi: 10.1002/hep.26314.

[65] Naeshiro N, Kakizawa H, Aikata H, Kan H, Fujino H, Fukuhara T, et al. Percutaneous transvenous embolization for portosystemic shunts associated with encephalopathy: Long-term outcomes in 14 patients. Hepatol Res 2014;44:740-749. doi: 10.1111/hepr.12181.

[66] Zidi SH, Zanditenas D, Gelu-Siméon M, Rangheard AS, Valla DC, Vilgrain V, et al. Treatment of chronic portosystemic encephalopathy in cirrhotic patients by embolization of portosystemic shunts. Liver Int 2007;27: 1389-1393. doi: 10.1111/j.1478-3231.2007.01602.x.

[67] Singh S, Kamath PS, Andrews JC, Leise MD. Embolization of spontaneous portosystemic shunts for management of severe persistent hepatic encephalopathy. Hepatology 2014;59:735-736. doi: 10.1002/hep.26575.
[68] Riggio O, Masini A, Efrati C, Nicolao F, Angeloni S, Salvatori FM, et al. Pharmacological prophylaxis of hepatic encephalopathy after transjugular intrahepatic portosystemic shunt: a randomized controlled study. J Hepatol 2005;42:674-679. doi: 10.1016/j.jhep.2004.12.028.

[69] Hassanein TI, Tofteng F, Brown RS Jr, McGuire B, Lynch P, Mehta R, et al. Randomized controlled study of extracorporeal albumin dialysis for hepatic encephalopathy in advanced cirrhosis. Hepatology 2007;46:1853-1862. doi: 10.1002/hep.21930.

[70] Campagna F, Montagnese S, Schiff S, Biancardi A, Mapelli D, Angeli P, et al. Cognitive impairment and electroencephalographic alterations before and after liver transplantation: what is reversible? Liver Transpl 2014;20: 977-986. doi: 10.1002/It.23909.

[71] Mechtcheriakov S, Graziadei IW, Mattedi M, Bodner T, Kugener A, Hinterhuber $\mathrm{HH}$, et al. Incomplete improvement of visuo-motor deficits in patients with minimal hepatic encephalopathy after liver transplantation. Liver Transpl 2004;10:77-83. doi: 10.1002/lt. 20009.

[72] Sotil EU, Gottstein J, Ayala E, Randolph C, Blei AT. Impact of preoperative overt hepatic encephalopathy on neurocognitive function after liver transplantation. Liver Transpl 2009;15:184-192. doi: 10.1002/It.21593.

[73] Garcia-Martinez R, Rovira A, Alonso J, Jacas C, Simón-Talero M, Chavarria L, et al. Hepatic encephalopathy is associated with posttransplant cognitive function and brain volume. Liver Transpl 2011;17:38-46. doi: 10.1002/It. 22197.

[74] Barritt AS 4th, Fried MW, Hayashi PH. Persistent portosystemic shunts after liver transplantation causing episodic hepatic encephalopathy. Dig Dis Sci 2010;55:1794-1798. doi: 10.1007/s10620-009-0901-6.

[75] Abujudeh HH, Samanta AK, Cho KC, Klein KM, Bahramipour P, Koneru B. Images in liver transplantation. Spontaneous portosystemic shunting several years following liver transplantation: successful treatment via percutaneous embolization. Liver Transpl 2004;10:324-326. doi: 10.1002/It. 20018.

[76] Rahimi RS, Rockey DC. Hepatic encephalopathy: pharmacological therapies targeting ammonia. Semin Liver Dis 2016;36:48-55. doi: 10.1055/s-00361571298. 